\title{
Norm Functions for Probabilistic Bisimulations with Delays
}

\author{
Christel Baier ${ }^{1}$ and Mariëlle Stoelinga ${ }^{2}$ \\ 1 Institut für Informatik I, Universität Bonn \\ Römerstraße 164, D-53117 Bonn, Germany \\ baier@cs . uni-bonn.de \\ 2 Computing Science Institute, University of Nijmegen \\ P.O.Box 9010, 6500 GL Nijmegen,The Netherlands \\ marielle@cs.kun.nl
}

\begin{abstract}
We consider action-labelled systems with non-deterministic and probabilistic choice. Using the concept of norm functions [17, we introduce two types of bisimulations (called (strict) normed bisimulation equivalence) that allow for delays when simulating a transition and are strictly between strong and weak bisimulation equivalence à la 26!36 37. Using a suitable modification of the prominent splitter/partitioning technique 2530, we present polynomial-time algorithms that constructs the quotient space of the (strict) normed bisimulation equivalence classes.
\end{abstract}

\section{Introduction}

Probabilistic aspects play a crucial role for a quantitative analysis of various types of parallel systems, such as systems that are designed on the basis of a randomized algorithms or computer systems with unreliable components. In the former case, probabilities can be used to specify the frequencies of the possible outcomes of an explicit probabilistic choice ("tossing a fair coin"); in the latter case, probabilities might express failure rates. Besides the probabilistic choices, the (transition) systems we consider allow for nondeterministic choices. These can be used for modelling probabilistic systems with asynchronous parallelism [41 19 18 18 34 5 |5 where the non-determinism is used to describe the interleaving of the subprocesses. Moreover, as observed by several authors [212334], the non-determinism can also be used to represent underspecification or incomplete information about the environment. Due to the combination of non-determinism and probability, the design and analysis of such systems (with both types of choices) can be hard.

Like for any kind of computer systems, the use of implementation relations (which compare two systems; thus yielding a formal definition of when a program $\mathcal{P}$ implements correctly another one $\mathcal{P}^{\prime}$ ) have turned out to be useful for the design and the system analysis. In this paper, we restrict to the equivalences that yield a notion of process equality. There are several highly desirable conditions that any reasonable process equivalence $\approx$ should fulfill, including e.g. the soundness for establishing quantitative linear time properties and congruence 
properties w.r.t. certain composition operators of a process calculus (such as parallel composition). A further crucial aspect is the development of methods that support the proof of the equivalence of two processes (i.e. deductive or algorithmic techniques to show $\mathcal{P} \approx \mathcal{P}^{\prime}$ ). The algorithmic methods are of great importance for automatic verification tools that take as their input a system $\mathcal{P}$ and its specification $\mathcal{P}^{\prime}$ and decides whether $\mathcal{P}$ correctly implements $\mathcal{P}^{\prime}$. Moreover, algorithms for computing the quotient space yield an abstraction technique which is highly relevant for the system analysis. For this, one replaces the states by their equivalence classes and then establishes the desired properties for the quotient space $S / \approx$ rather than the original state space $S$. Especially when we deal with weak equivalences (that abstract from internal computations) the switch from the original system $S$ to the quotient space $S / \approx$ might lead to a much smaller equivalent system; and hence can be viewed as a technique to combat the state explosion problem.

Several (strong and weak) equivalences for various types of probabilistic systems have been proposed in the literature. They range over the full linear and branching time spectrum and are extensions of the corresponding relations on LTSs. While in the fully probabilistic setting, the equivalences are studied under several aspects (compositionality, axiomatization, decidability, logical characterizations, etc.), see e.g. 24,10,22 20]27,94, the treatment of equivalences for probabilistic systems with non-determinism is less well-understood. Most of the standard relations that have proven to be useful in the non-probabilistic setting have been extended for the probabilistic case; see e.g. [35] for a trace-based relation, 42 23] for testing equivalences and [26 19 18 36 43 3735 38 39] for several types of (bi-)simulations. However, due to the combination of non-determinism and probability, the definitions are more complicated than the corresponding notions for non-probabilistic or fully probabilistic systems. Even though some important issues (like compositionality and axiomatization) have been addressed in the above mentioned literature, research on algorithmic methods to decide the equivalence of two systems or to compute the quotient space are rare. For strong bisimulation [26] and strong simulation [36], polynomial-time algorithms have been presented in [3]. To the best of our knowledge, the forthcoming work [32] is the first attempt to formulate an algorithmic method that deals with a weak equivalence for probabilistic processes with non-determinism. We are not aware of any complexity (or even decidability) result for weak bisimulation à la [36 37] or any linear time relation on probabilistic systems with non-determinism, e.g. trace distribution equivalence [35] 1 ]

Our contribution: We deal with probabilistic systems with non-determinism and action labels modelled by a probabilistic extension of LTSs where the (actionlabelled) transitions are augmented with probabilities for the possible target states. Our model essentially agrees with the simple probabilistic automata of

${ }^{1}$ As (non-probabilistic) LTSs are special instances of probabilistic systems with nondeterminism and the trace distribution preorder à la Segala is a conservative extension of usual trace containment, the PSPACE-completeness for LTSs 25] yields the PSPACE-hardness for the trace distribution relation à la [35]. 
[36 34]). Our main contribution is the presentation of novel notions of bisimulation equivalence which (in some sense) are insensitive with respect to internal transitions. More precisely, our equivalences are conservative extensions of delay bisimulation equivalence [4014] which relies on the assumption that the simulation of a step of a process $\mathcal{P}$ by another process $\mathcal{P}^{\prime}$ might happen with a certain delay (i.e. after a sequence of internal transitions). The formal definition of our equivalences is provided by a probabilistic variant of norm functions in the style of [17]. Intuitively, the norm functions specify bounds for the delays (i.e. the number of internal transitions that might be performed before a "proper" transition of a process $\mathcal{P}$ is simulated by a corresponding transition of an equivalent process $\mathcal{P}^{\prime}$ ). In the probabilistic setting where the combination of internal transitions leads to a tree rather than a linear chain, the norm functions yield conditions on the length of the paths in the trees corresponding to a "delayed transition". Using a modification of the traditional splitter/partioning technique [25,30], we present polynomial time algorithms for computing the quotient spaces. Moreover, we briefly discuss some other aspects (compositionality w.r.t. parallel composition and preservation of linear time properties).

Organization of the paper: Section 2 introduces our model for probabilistic labelled transition systems. The definitions of norm functions and normed bisimulations are presented in Section 3 In Section 4 we present our algorithm for computing the bisimulation equivalence classes. Section 5 concludes the paper.

Because of space restrictions, we present our main results without proofs. We refer the interested reader to [6] where the proofs and other details (including results about various types of bisimulations and simulations) can be found.

\section{Probabilistic Labelled Transition Systems}

In (ordinary) LTSs, the transitions $s \stackrel{a}{\longrightarrow} t$ specify the possibility that the system in state $s$ moves via the action $a$ to state $t$. In this paper, we deal with a probabilistic variant of LTSs where any transition is augmented with a probabilistic choice for the possible target states (rather than a unique target state $t$ as it is the case in LTSs). That is, in the probabilistic setting, the transitions are of the form $s \stackrel{a}{\longrightarrow} \mu$ where $s$ is the starting state, $a$ an action label and $\mu$ a distribution on the state space which specifies the probabilities $\mu(t)$ for any possible successor state $t$. Non-determinism is present in our model since we allow several (possibly equally action-labelled) outgoing transitions of a state $s$.

Notation 1 Let $S$ be a finite set. A distribution on $S$ is a function $\mu: S \rightarrow[0,1]$ such that $\sum_{s \in S} \mu(s)=1$. Let $\operatorname{Supp}(\mu)=\{s \in S: \mu(s)>0\}$ denote the support of $\mu ; \mu[A]=\sum_{s \in A} \mu(s)$ for $\emptyset \neq A \subseteq S$ and $\mu[\emptyset]=0$. For $s \in S, \mu_{s}^{1}$ denotes the unique distribution on $S$ with $\mu_{s}^{1}(s)=1$. $\operatorname{Distr}(S)$ denotes the collection of all distributions on $S$. If $R$ is an equivalence relation on $S$ then $S / R$ to denotes the quotient space of $S$ with respect to $R$. The induced equivalence $\equiv_{R}$ on $\operatorname{Distr}(S)$ is given by $\mu \equiv_{R} \mu^{\prime}$ iff $\mu[A]=\mu^{\prime}[A]$ for all $A \in S / R$. We write $[\mu]_{R}$ for the equivalence class $\left\{\mu^{\prime}: \mu \equiv_{R} \mu\right\}$ of $\mu$ with respect to $\equiv_{R}$. 
Definition 1. A probabilistic labelled transition system (PLTS for short) is a tuple $(S, A c t, \longrightarrow)$ where $S$ is a finite set of states, Act a finite set of actions (containing a special symbol $\tau$ ) 2 and $\longrightarrow \subseteq S \times A c t \times \operatorname{Distr}(S)$ a transition relation such that for all $s \in S$ and $a \in A c t, \operatorname{Steps}_{a}(s)=\{\mu: s \stackrel{a}{\longrightarrow} \mu\}$ is finite 3 A probabilistic program is a tuple $\mathcal{P}=\left(S, A c t, \longrightarrow, s_{\text {init }}\right)$ consisting of a PLTS $(S, A c t, \longrightarrow)$ and an initial state $s_{\text {init }} \in S$.

Example 1. We consider a simple communication protocol consisting of a sender (that produces certain messages and tries to submit the messages along an unreliable medium) and a receiver (that acknowledges the receipt and consumes the received messages). For simplicity, we assume that both the sender and the receiver work with mailing boxes that cannot hold more than one message at any time. The failure rate of the medium is $1 \%$; i.e., with probability $1 / 100$ the medium looses the messages and the sender retries to submit the message. In state $s_{\text {init }}$, the sender produces a message and passes the message to the medium which leads to the state $s_{\text {del }}$ (where the medium tries to deliver the message via an internal action). When the message is delivered correctly, the state $s_{o k}$ is reached. In state $s_{o k}$, the sender and the receiver can work in parallel (modelled by interleaving): the sender may produce the next message while the receiver may consume the last message.

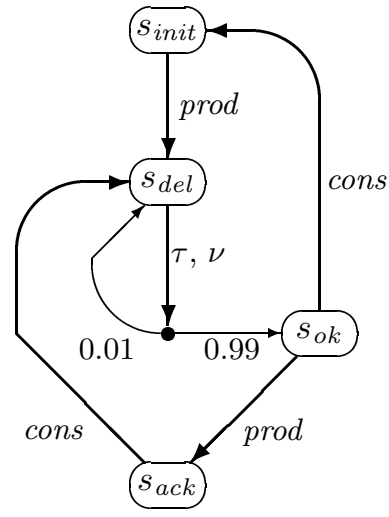

The executions of a PLTS are given by the paths in the underlying directed graph. They arise through the resolution of both the non-deterministic and probabilistic choices 4 Typically, one assumes that the resolution of the nondeterministic choices are not under the control of the system itself. The entity that resolves the non-determinism (the "environment") can be formalized by a scheduler [41] (also called adversary [34] or policy in the theory of MDPs [33]). Given a scheduler $A$, the system behaviour under $A$ can be described by a Markov chain which yields a Borel field and probability measure on the paths that can be obtained by $A$. The details are not of importance for this paper and are omitted here. They can be found e.g. in the above mentioned references.

\section{Normed Bisimulation}

In ordinary LTSs, the several types of bisimulations (e.g. strong, weak branching or delay bisimulation 2831,29 164014) establish a correspondence between the

${ }^{2}$ We refer to $\tau$ as the internal action. All other actions are called visible.

${ }^{3}$ Any finite $\operatorname{LTS}(S, A c t, \longrightarrow)$ (where $\left.\longrightarrow \subseteq S \times A c t \times S\right)$ can be viewed as a PLTS. For this, we identify any transition $s \stackrel{a}{\longrightarrow} t$ with its probabilistic counterpart $s \stackrel{a}{\longrightarrow} \mu_{t}^{1}$.

${ }^{4}$ Formally, a path is a "sequence" $s_{0} \stackrel{a_{1}, \mu_{1}}{\longrightarrow} s_{1} \stackrel{a_{2}, \mu_{2}}{\longrightarrow} s_{2} \stackrel{a_{2}, \mu_{2}}{\longrightarrow} \ldots$ where $s_{i-1} \stackrel{a_{i}}{\longrightarrow} \mu_{i}$, $s_{i} \in \operatorname{Supp}\left(\mu_{i}\right)$. 
states and their stepwise behaviour. Intuitively, they identify those states $s$ and $s^{\prime}$ where any outgoing transition from $s$ can be simulated by $s^{\prime}$ and vice versa. Most types of bisimulation equivalences on a $\operatorname{LTS}(S$, Act,$\longrightarrow)$ can be characterized as the coarsest equivalence $R$ on the state space $S$ such that

(Bis) If $\left(s, s^{\prime}\right) \in R, C \in S / R$ and $s \stackrel{a}{\longrightarrow} C$ then $s^{\prime} \in \operatorname{Pre}^{*}(a, C)$.

Here, we write $s \stackrel{a}{\longrightarrow} C$ if $s \stackrel{a}{\longrightarrow} t$ for some $t \in C$. Pre ${ }^{*}(a, C)$ denotes a certain predecessor predicate. Intuitively, $s^{\prime} \in \operatorname{Pre}^{*}(a, C)$ asserts that $s^{\prime}$ can "simulate" the transition $s \stackrel{a}{\longrightarrow} C$. The formal definition of $\operatorname{Pre}^{*}(a, C)$ depends on the concrete type of equivalence. E.g., strong bisimulation is obtained by using the predicate $\operatorname{Pre}^{\text {sbis }}(a, C)=\left\{s^{\prime}: s^{\prime} \stackrel{a}{\longrightarrow} C\right\}$ while delay bisimulation equivalence [4014] focuses on the idea that the simulation of a transition $s \stackrel{a}{\longrightarrow} t$ might happen with a certain delay (i.e. after a finite number of internal moves) and uses the predicates $\operatorname{Pre}^{d e l}(\cdot)$ which are given by the following three conditions. 5

(D0) $C \subseteq \operatorname{Pre}^{\mathrm{del}}(\tau, C)$

(D1) If $s \stackrel{a}{\longrightarrow} C$ then $s \in \operatorname{Pre}^{\operatorname{del}}(a, C)$.

(D2) If $s \stackrel{\tau}{\longrightarrow} t$ and $t \in \operatorname{Pre}^{d e l}(a, C)$ then $s \in \operatorname{Pre}^{d e l}(a, C)$.

[26] presented an elegant reformulation of strong bisimulation for a variant of PLTSs which takes the probabilistic effect of the transitions into account. Formally, strong bisimulation equivalence $\approx_{s b i s}$ in a PLTSs is the coarsest equivalence $R$ on the state space $S$ such that for all $\left(s, s^{\prime}\right) \in R$ and transitions $s \stackrel{a}{\longrightarrow} \mu$ there is a transition $s^{\prime} \stackrel{a}{\longrightarrow} \mu^{\prime}$ where $\mu$ and $\mu^{\prime}$ return the same probabilities for all equivalence classes under $R$ (i.e. $\mu \equiv_{R} \mu^{\prime}$, cf. Notation [1). 3637] presented notions of weak and branching bisimulations for PLTSs. All these notions of bisimulation equivalences on a PLTS $(S, A c t, \longrightarrow)$ can be characterized as the coarsest equivalence $R$ on $S$ such that

(PBis) If $\left(s, s^{\prime}\right) \in R, M \in \operatorname{Distr}(S) / \equiv_{R}$ and $s \stackrel{a}{\longrightarrow} M$ then $s^{\prime} \in \operatorname{Pre}^{*}(a, M)$. Here, $s \stackrel{a}{\longrightarrow} M$ iff $s \stackrel{a}{\longrightarrow} \mu$ for some $\mu \in M$. E.g., strong bisimulation equivalence is given by (PBis) using the predecessor predicate $\operatorname{Pre}{ }^{\text {sbis }}(a, M)=\left\{s^{\prime}: s^{\prime} \stackrel{a}{\longrightarrow} M\right\}$.

We now propose novel notions of bisimulation equivalence for PLTSs which are conservative extensions of delay bisimulation equivalence 4014. Intuitively, two states $s, s^{\prime}$ are identified iff any transition $s \stackrel{a}{\longrightarrow} \mu$ can be simulated by $s^{\prime}$ by first performing finitely many internal moves and then performing an $a$ labelled transition for which the outcome of the associated probabilistic choice agrees with $\mu 6$ Thus, we aim at an appropriate definition of the predecessor predicate $\operatorname{Pre}^{d e l}(a, M)$ where (for $a \neq \tau$ or $\left.\mu_{s^{\prime}}^{1} \notin M\right) s^{\prime} \in \operatorname{Pre}^{d e l}(a, M)$ states the possibility for $s^{\prime}$ to perform the action $a$ (possibly with a certain delay) such that the associated distribution $\mu^{\prime}$ of the $a$-labelled transition belongs to $M=[\mu]_{R}$. Conditions (D0) and (D1) for $\operatorname{Pre}^{d e l}(a, C)$ can easily be lifted to the probabilistic case (see conditions (BD0) and (BD1) below).

${ }^{5}$ Thus, $\operatorname{Pre}^{d e l}(a, C)=\left\{s^{\prime}: s^{\prime} \stackrel{\tau^{*} a}{\longrightarrow} C\right\}$ for $a \neq \tau$ and $\operatorname{Pre}^{d e l}(\tau, C)=\left\{s^{\prime}: s^{\prime} \stackrel{\tau^{*}}{\longrightarrow} C\right\}$.

${ }^{6}$ This informal explanation assumes that $s \stackrel{a}{\longrightarrow} \mu$ is a "proper" transition, i.e. either $a \neq \tau$ or $\mu_{s}^{1} \notin M$. Transitions of the form $s \stackrel{\tau}{\longrightarrow} \mu$ where all possible target states $t \in \operatorname{Supp}(\mu)$ are equivalent to $s$ can be viewed as "silent moves" and are not taken account when dealing with equivalences that abstract from internal computations. 
(BD0) If $\mu_{s}^{1} \in M$ then $s \in \operatorname{Pre}^{\text {del }}(\tau, M)$.

(BD1) If $s \stackrel{a}{\longrightarrow} M$ then $s \in \operatorname{Pre}^{\text {del }}(a, M)$.

To adapt condition (D2) for the probabilistic setting, we have two possibilities depending on whether or not we allow for unbounded delays. For the simpler case, we require bounded delays which leads to condition (BD2).

(BD2) If $s \stackrel{\tau}{\longrightarrow} \nu$ and $\operatorname{Supp}(\nu) \subseteq \operatorname{Pre}^{d e l}(a, M)$ then $s \in \operatorname{Pre}^{d e l}(a, M)$.

The resulting bisimulation equivalence only abstracts from the combination of finitely many internal moves (corresponding to a bounded delay) but cannot involve the effect of infinite $\tau$-paths (unbounded delays). In the communication protocol of Example 1 one might argue that the states $s_{d e l}$ and $s_{o k}$ have the same observable behaviour as $s_{\text {del }}$ moves via $\tau$-transitions to $s_{o k}$ with probability 1. To formalize the effect of infinite $\tau$-loops, we use the concept of norm functions which was introduced in [17] to reason about simulation-like relations in nonprobabilistic systems. We slightly depart from the notations of [17] and define norm functions in LTSs as partial functions with three arguments (a state $s$, an action label $a$ and a set $C$ of target states) and whose range are the natural numbers. If the value $n(s, a, C)$ is defined then $s \in \operatorname{Pre}^{d e l}(a, C)$ in which case there is a $\tau^{*}$-labelled path of length $\leq n(s, a, C)$ from $s$ to a state $t$ where either $t \stackrel{a}{\longrightarrow} C$ or $a=\tau$ and $t \in C$. If $s \notin \operatorname{Pre}^{\operatorname{del}}(a, C)$ then $n(s, a, C)$ is undefined (denoted $n(s, a, C)=\perp$ ). The formal definition of norm functions in LTSs arises by "refining" the above mentioned three conditions for $\operatorname{Pre}^{d e l}(a, C)$ in the sense that we involve the length of a delayed transition. Formally, norm functions in LTSs are partial functions satisfying the following three conditions.

(N0) $n(s, a, C)=0$ implies $a=\tau$ and $s \in C$

(N1) $n(s, a, C)=1$ implies $s \stackrel{a}{\longrightarrow} C$

(N2) If $n(s, a, C) \geq 2$ then there is a transition $s \stackrel{\tau}{\longrightarrow} t$ where $n(t, a, C)<$ $n(s, a, C)$.

To adapt these three conditions to the probabilistic setting, we deal with a set $M \subseteq \operatorname{Distr}(S)$ as the third argument of a norm function. The modifications of $(\mathrm{N} 0)$ and (N1) are straightforward. In (N2) we require that $n(s, a, M) \geq 2$ implies the existence of a transition $s \stackrel{\tau}{\longrightarrow} \nu$ satisfying a certain condition. When we aim at bounded delays then we deal with the constraint $n(t, a, M)<n(s, a, M)$ for all $t \in \operatorname{Supp}(\nu)$. For unbounded delays, we require that $n(t, a, M)$ is defined for all $t \in \operatorname{Supp}(\nu)$ and $n(t, a, M)<n(s, a, M)$ for some $t \in \operatorname{Supp}(\nu)$ ]

Definition 2. A norm function for a PLTS $(S, A c t, \longrightarrow)$ is a partial function $n: S \times A c t \times 2^{\operatorname{Distr}(S)} \rightarrow \mathbb{N}$ which satisfies the following conditions.

(PN0) $n(s, a, M)=0$ implies $a=\tau$ and $\mu_{s}^{1} \in M$.

(PN1) $n(s, a, M)=1$ implies $s \stackrel{a}{\longrightarrow} M$ (i.e. $s \stackrel{a}{\longrightarrow} \mu$ for some $\mu \in M$ ).

\footnotetext{
7 These two conditions about $\operatorname{Supp}(\nu)$ guarantee the existence of a scheduler where, for any state $s$ for which $n(s, a, M)$ is defined, almost all paths starting in $s$ lead via $\tau$ 's to a state $t$ where $n(t, a, M) \in\{0,1\}$. Thus, in this scheduler, with probability 1 , $s$ performs finitely many $\tau$ 's followed by a transitions $t \stackrel{a}{\longrightarrow} \mu^{\prime}$ where $\mu^{\prime} \in M$. However, for this scheduler, there might be no upper bound for the number of $\tau$ 's that will be performed before the action $a$.
} 
(PN2) If $n(s, a, M) \geq 2$ then there is a transition $s \stackrel{\tau}{\longrightarrow} \nu$ where

(i) $n(t, a, M) \neq \perp$ for all $t \in \operatorname{Supp}(\nu)$

(ii) $n(t, a, M)<n(s, a, M)$ for some $t \in \operatorname{Supp}(\nu)$

$n$ is strict iff, in (ii), $n(t, a, M)<n(s, a, M)$ for all $t \in \operatorname{Supp}(\nu)$.

Example 2. Consider the system on the right. Let $M$ be the set of distributions $\mu$ that return probability 1 for the $x$-states (i.e. $M=$ $\left.\left\{\mu: \mu\left(x_{1}\right)+\mu\left(x_{2}\right)=1\right\}\right)$. Then, $\mu_{x_{1}}^{1}, \mu_{x_{2}}^{1} \in$ $M$ and $s_{1} \stackrel{a}{\longrightarrow} M, s_{2} \stackrel{a}{\longrightarrow} M$. Thus, the partial function $n$ with $n\left(s_{0}, a, M\right)=2, n\left(s_{1}, a, M\right)$ $=n\left(s_{2}, a, M\right)=1$ and $n(\cdot)=\perp$ in all other cases is a strict norm function.

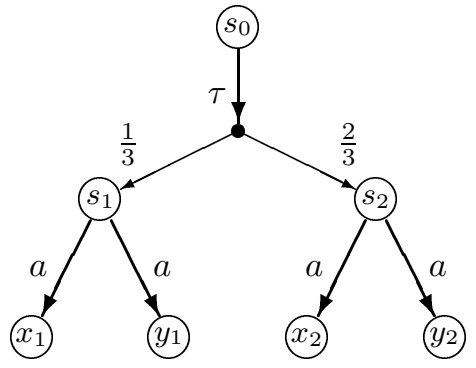

Definition 3. Let $(S, A c t, \longrightarrow)$ be a PLTS and $R$ an equivalence on $S . R$ is called a (strict) normed bisimulation iff there exists a (strict) norm function $n$ such that for all $a \in A c t$ and $M \in \operatorname{Distr}(S) / \equiv_{R}$ : if $\left(s, s^{\prime}\right) \in R$ and $s \stackrel{a}{\longrightarrow} M$ then $n\left(s^{\prime}, a, M\right) \neq \perp$. Two states $s$ and $s^{\prime}$ are called (strictly) normed bisimilar (denoted $s \approx_{n} s^{\prime}$ resp. $s \approx_{s n} s^{\prime}$ ) iff there exists a (strict) normed bisimulation $R$ such that $\left(s, s^{\prime}\right) \in R$. The equivalences $\approx_{n}$ and $\approx_{s n}$ are adapted for probabilistic programs in the obvious way 8

Example 3. It is easy to see that the states $s_{0}$, $s_{1}$ and $s_{2}$ in Example 7 are strictly normed bisimilar. For the simple communication protocol of Example 1 and the smallest equivalence relation $R$ that identifies $s_{d e l}$ and $s_{o k}$, there is a norm function with $n\left(s_{o k}, \tau,[\nu]_{R},\right)=0$ and $n\left(s_{\text {del }}\right.$, cons,$\left.\left[s_{\text {init }}\right]_{R}\right)=2$ but no strict norm function. Thus, $s_{o k} \approx_{n} s_{d e l}$ but $s_{o k} \not_{s n} s_{d e l}$. The quotient system that we get when we identify the states by their normed bisimulation equivalence classes can be viewed as a failure-free specification (see the picture on the right).

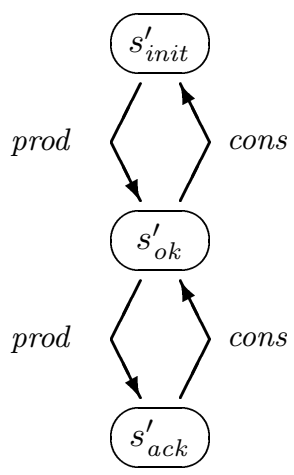

$\approx_{n}$ and $\approx_{s n}$ can be characterized by condition (PBis) with suitable defined predecessor predicates. The unbounded delay predecessor predicate $\operatorname{Pre}_{u b}^{d e l}(a, M)$ is the set of states $s$ where $n(s, a, M) \neq \perp$ for some norm function $n$. The bounded predecessor predicate $\operatorname{Pre}_{b}^{d e l}(a, M)$ is the set of states $s$ such that $n(s, a, M) \neq \perp$ for some strict norm function $n 9$ Then, $\operatorname{Pre}_{b}^{d e l}(a, M)$ is the least set satisfying the three conditions (BD0), (BD1), (BD2). In what follows,

${ }^{8}$ Recall that a probabilistic program is a PLTS with an initial state (Def. 四). Let $\mathcal{P}_{i}$ be probabilistic programs with initial states $s_{i}, i=1,2$. We define $\mathcal{P}_{1} \approx_{*} \mathcal{P}_{2}$ iff $s_{1} \approx_{*} s_{2}$ where $s_{1}, s_{2}$ are viewed as states in the composed system $\mathcal{P}_{1} \uplus \mathcal{P}_{2}$ which arises from the disjoint union of the state spaces of $\mathcal{P}_{1}$ and $\mathcal{P}_{2}$.

${ }^{9}$ For a LTS, viewed as a PLTS, the unbounded and bounded predecessor predicates coincide. More precisely, $\operatorname{Pre}^{d e l}(a, C)=\operatorname{Pre}_{u b}^{d e l}\left(a, M_{C}\right)=\operatorname{Pre}_{b}^{d e l}\left(a, M_{C}\right)$ for any set $C$ of states and $M_{C}=\left\{\mu_{t}^{1}: t \in C\right\}$. 
we simply write $\operatorname{Pre}^{d e l}(a, M)$ to denote $\operatorname{Pre}_{b}^{d e l}(a, M)$ or $\operatorname{Pre}_{u b}^{d e l}(a, M)$ depending on whether we deal with strict normed bisimulation or normed bisimulation equivalence. It is easy to see that (strict) normed bisimulation equivalence meets the general characterization of bisimulation equivalences in PLTSs via condition (PBis). More precisely, (strict) normed bisimulation equivalence is the coarsest equivalence $R$ on $S$ such that $\left(s, s^{\prime}\right) \in R, M \in \operatorname{Distr}(S) / \equiv_{R}$ and $s \stackrel{a}{\longrightarrow} M$ implies $s^{\prime} \in \operatorname{Pre}^{d e l}(a, M)$. (Strict) normed bisimulation equivalence lies strictly between strong $\left(\approx_{s b i s}\right)$ and weak $\left(\approx_{w b i s}\right)$ bisimulation equivalence à la 263637, i.e. $\approx_{\text {sbis }} \subset \approx_{s n} \subset \approx_{n} \subset \approx_{\text {wbis }}$. The communication protocol and its failure free specification are examples that demonstrate the difference between $\approx_{s n}$ and $\approx_{n}$.

A crucial property of a simulation equivalence is soundness w.r.t. parallel composition, since this allows for compositional analysis. Another very important property is soundess w.r.t. a specification logic. For divergent-free processes (processes without $\tau$-loops), our equivalences are sound for quantitive linear time properties. These express that, independent how the nondeterminism is resolved, the probability on a certain set of traces is larger than some number $p$.

Proposition 1. If $\mathcal{P}_{1}$ and $\mathcal{P}_{2}$ are divergence free and $\mathcal{P}_{1} \approx_{n} \mathcal{P}_{2}$, then $\mathcal{P}_{1}$ and $\mathcal{P}_{2}$ satisfy exactly the same quantative lineair time properties.

Proposition 2. $\mathcal{P}_{1} \approx_{n} \mathcal{P}_{2}$ implies $\mathcal{P}_{1}\left\|\mathcal{Q} \approx_{n} \mathcal{P}_{2}\right\| \mathcal{Q}$ and similarly for $\approx_{s n}$.

\section{Decidability}

In this section, we present an algorithm that computes the (strict) normed bisimulation equivalence classes in polynomial time and space. The main idea of our algorithm is a modification of the prominent splitter/partitioning technique [2530] (which is sketched in Figure 1) that was proposed for computing the strong bisimulation equivalence classes in a non-probabilistic transition system. The basic idea is to start with the trivial partition $\chi=\{S\}$ of the state space $S$ and then successively refine $\chi$ by splitting the blocks $B$ of $\chi$ into subblocks according to a refinement operator $\operatorname{Ref}(\chi, a, C)$ that depends on a splitter, i.e. an action/block pair $\langle a, C\rangle$. More precisely, $\operatorname{Ref}(\chi, a, C)$ divides each block $B \in \chi$ into the subblocks $B \cap \operatorname{Pre}^{\text {str }}(a, C)$ and its complement $B \backslash \operatorname{Pre}^{\text {str }}(a, C)$. 10 Using an appropriate organization of the splitters (resp. splitter candidates), this method can be implemented in time $\mathcal{O}(m \log n)$ where $n$ is the number of states and $m$ the number of transitions (i.e. the size of $\longrightarrow$ ) [30]. The above sketched technique can easily be modified to compute several other types of bisimulation equivalence classes, such as the strong [20] or weak [4] bisimulation equivalence classes in fully probabilistic systems, but fails for strong (and hence for normed) bisimulation in PLTSs when action/block pairs are used as splitters [3].

${ }^{10} \operatorname{Ref}(\chi, a, C)$ yields the partition $\bigcup_{B \in \chi} \operatorname{Ref}(B, a, C)$ where $\operatorname{Ref}(B, a, C)=\{B \cap$ $\left.\operatorname{Pre}^{s t r}(a, C), B \backslash \operatorname{Pre}^{\text {str }}(a, C)\right\} \backslash\{\emptyset\}$. 
$\chi:=\{S\}$

While $\chi$ can be refined do

choose some splitter $\langle a, C\rangle$ of $\chi$ and put $\chi:=\operatorname{Ref}(\chi, a, C)$;

Return $\chi$.

Fig. 1. Schema for computing the bisimulation equivalence classes in LTSs

In the remainder of this section, we explain how the splitter/partitioning technique can be modified to get a polynomial-time algorithm for computing the (strict) normed bisimulation equivalence classes in a PLTS.

Notation 2 We fix a PLTS $(S$, Act, $\longrightarrow)$. Let $M_{a}=\bigcup_{s \in S}$ Steps $_{a}(s)$. For $Z$ to be a finite set, we write $|Z|$ to denote the number of elements in $Z$. Let $n=|S|$ the number of states, $m=|\longrightarrow|$ the total number of transitions and $m_{\tau}=\sum_{s \in S} \mid$ Steps $_{\tau}(s) \mid$ the number of $\tau$-transitions. We assume that Act does not contain redundant actions, i.e. we require that $M_{a} \neq \emptyset$ for all actions $a$.

We use similar ideas as suggested in 3] where an algorithm for computing the strong bisimulation equivalence classes of a PLTS in time $\mathcal{O}(m n(\log m+\log n))$ is presented. The key idea is to refine the current state partition has be according to splitters of the form $\langle a, M\rangle$ where $a$ is an action and $M$ a subset of $M_{a}$. That is, we successively replace the current state partition $\chi$ by

$$
\operatorname{Ref}(\chi, a, M)=\bigcup_{B \in \chi} \operatorname{Ref}(B, a, M)
$$

where $\operatorname{Ref}(B, a, M)=\left\{B \cap \operatorname{Pre}^{\text {del }}(a, M), B \backslash \operatorname{Pre}^{\text {del }}(a, M)\right\} \backslash\{\emptyset\}$.

Notation 3 A step partition is a set $\mathcal{M}$ consisting of pairs $\langle a, M\rangle$ where $M \subseteq$ $M_{a}$ and such that, for any action $a,\{M:\langle a, M\rangle \in \mathcal{M}\}$ is a partition of $M_{a}$. We refer to the pairs $\langle a, M\rangle$ as step classes. Given a state partition $\chi$, the induced step partition $\mathcal{M}_{\chi}$ consists of the step classes $\langle a, M\rangle$ where $M \in M_{a} / \equiv_{\chi}$ and $\mu \equiv_{\chi} \mu^{\prime}$ iff $\mu[C]=\mu^{\prime}[C]$ for all $C \in \chi$.

$\chi:=\{S\}$

While $\chi$ can be refined do

choose some step class $\langle a, M\rangle$ of $\mathcal{M}_{\chi}$ and put $\chi:=\operatorname{Ref}(\chi, a, M)$;

Return $\chi$.

Fig. 2. Schema for computing the bisimulation equivalence classes in PLTSs

The rough ideas behind our algorithm are sketched in Figure 2. To keep book about the splitter candidates $\langle a, M\rangle$ we use a step partition $\mathcal{M}$ (that agrees with $\mathcal{M}_{\chi}$ after any iteration) and a set $\operatorname{SplCnd}$ (e.g. organized as a queue) which contains the step classes that will serve as splitter candidates. Initially, 
SplCnd consists of the "trivial" step classes $\left\langle a, M_{a}\right\rangle$. In each iteration, we first refine the state partition $\chi$ according to a step class $\langle a, M\rangle \in S p l C n d$ which yields the new state partition $\chi_{\text {new }}=\operatorname{Ref}(\chi, a, M)$. Then, we adjust $\mathcal{M}$ to $\chi_{\text {new }}$, i.e. calculate $\mathcal{M}_{\text {new }}=\mathcal{M}_{\chi_{\text {new }}}$. All new step classes $\left\langle b, N^{\prime}\right\rangle \in \mathcal{M}_{\text {new }} \backslash \mathcal{M}$ are viewed as splitter candidates and are inserted into $S p l C n d$. To derive $\mathcal{M}_{\text {new }}$ from $\mathcal{M}$ we have to replace any step class $\langle b, N\rangle$ in $\mathcal{M}$ by the step classes $\left\langle b, N^{\prime}\right\rangle$ where $N^{\prime} \in N / \equiv_{\chi}$. At the beginning of any iteration we have $\mathcal{M}=\mathcal{M}_{\chi}$. Thus, for $\langle b, N\rangle \in \mathcal{M}$ and $\nu, \nu^{\prime} \in N$ we have $\nu[B]=\nu^{\prime}[B]$ for all $B \in \chi$. Let $B \in \chi$ and $B^{\prime}=B \cap \operatorname{Pre}^{\text {del }}(a, M), B^{\prime \prime}=B \backslash B^{\prime}$ and $C^{\prime} \in\left\{B^{\prime}, B^{\prime \prime}\right\}$, $\langle b, N\rangle \in \mathcal{M}$ and $\nu, \nu^{\prime} \in N$. Then, $\nu\left[C^{\prime}\right]=\nu^{\prime}\left[C^{\prime}\right]$ iff $\nu\left[B^{\prime}\right]=\nu^{\prime}\left[B^{\prime}\right]$ and $\nu\left[B^{\prime \prime}\right]=$ $\nu^{\prime}\left[B^{\prime \prime}\right]$. These observations motivate the use of a set $N e w B l$ which contains only those blocks $C^{\prime} \in \chi_{\text {new }}$ that are relevant for the computation of $\mathcal{M}_{\text {new }}$. More precisely, for any block $B \in \chi$ where $|\operatorname{Ref}(B, a, M)|=2$, we choose a block $C_{B}^{\prime} \in \operatorname{Ref}(B, a, M)$ such that $\left|C_{B}^{\prime}\right| \leq|B| / 2$ and define $N e w B l=\left\{C_{B}^{\prime}: B \in\right.$ $\chi,|\operatorname{Ref}(B, a, M)|=2\}$. Then, $\mathcal{M}_{\text {new }}$ can be derived from $\mathcal{M}$ by replacing any $\langle b, N\rangle$ in $\mathcal{M}$ by the step classes in $\operatorname{Split}(\langle b, N\rangle, N e w B l)$ which we compute as follows. We start with $\mathcal{N}=\{\langle b, N\rangle\}$; Then, for all $C^{\prime} \in N e w B l$ we replace any $\left\langle b, N^{\prime}\right\rangle$ in $\mathcal{N}$ by $\operatorname{Split}\left(\left\langle b, N^{\prime}\right\rangle, C^{\prime}\right)$ where the operator $\operatorname{Split}\left(\left\langle b, N^{\prime}\right\rangle, C^{\prime}\right)$ divides $\left\langle b, N^{\prime}\right\rangle$ into the step classes $\left\langle b, N_{1}^{\prime}\right\rangle, \ldots,\left\langle b, N_{r}^{\prime}\right\rangle$ where $N_{1}^{\prime}, \ldots, N_{r}^{\prime}$ is the splitting of $N^{\prime}$ according to the probabilities for $C^{\prime} 11$ These ideas lead to the algorithm sketched in Figure 3 .

Theorem 1. The (strict) normed bisimulation equivalence classes can be computed in time $\mathcal{O}\left(m n(\log m+\log n)+m_{\tau} n^{2}\right)$ and space $\mathcal{O}(m n)$.

The remainder of this section is concerned with the proof of Theorem11. It follows from Prop. 3 and Prop. 4 We put $\chi_{0}=\{S\}$ and write $\chi_{i}$ to denote the state partition $\chi$ after the $i$-th iteration. Similarly, we use the notations $\mathcal{M}_{i}, \operatorname{SplCnd}_{i}$ and $\mathrm{NewBl}_{i}$ with the obvious meaning. Let AllSplCnd $=\bigcup_{i \geq 0} \operatorname{SplCnd}_{i}$ the set of all step classes $\langle a, M\rangle$ that once serve as splitters for the state partition $\chi$ and let $A l l N e w B l=\bigcup_{i} N e w B l_{i}$ the set of all blocks $C^{\prime}$ that once are used in a splitting operation $\operatorname{Split}\left(\cdot, C^{\prime}\right)$. Using set-theoretic arguments, we get:

(i) $\mid$ AllSplCnd $|\leq| \mathcal{M}_{0} \cup \mathcal{M}_{1} \cup \ldots \mid \leq 2(m-1)$

(ii) $\mid$ AllNewBl $|\leq| \chi_{0} \cup \chi_{1} \cup \ldots \mid \leq 2(n-1)$

(iii) $\sum_{C^{\prime} \in A l l N e w B l}\left|C^{\prime}\right| \leq n \log n$.

Proposition 3. The operations $\operatorname{Ref}(\chi, a, M)$ in step (2) of the algorithm in Fig. 3 can be implemented in time $\mathcal{O}\left(m_{\tau} n^{2}\right)$ (where we range over all iterations).

Proof. Clearly, given the predecessor predicate $\operatorname{Pre}^{\text {del }}(a, M)$ for a fixed step class $\langle a, M\rangle, \operatorname{Ref}(\chi, a, M)$ can be performed in time $\mathcal{O}(n)$ when appropriate data structures are used. Combining (i) and the following Lemmatas 1 and 2 we get the desired bound for the time complexity.

${ }^{11} \operatorname{Split}(\langle b, N\rangle, N e w B l)$ returns the set of step classes $\left\langle b, N^{\prime}\right\rangle$ where $N^{\prime} \in N / \equiv$ and $\nu \equiv \nu^{\prime}$ iff $\nu\left[C^{\prime}\right]=\nu^{\prime}\left[C^{\prime}\right]$ for all $C^{\prime} \in N e w B l$. As $N \in M_{b} / \equiv_{\chi}$ yields that $\equiv$ and $\equiv_{\chi_{\text {new }}}$ coincide, we get $\mathcal{M}_{\chi_{\text {new }}}=\bigcup_{\langle b, N\rangle \in \mathcal{M}} \operatorname{Split}(\langle b, N\rangle, N e w B l)$. 
$\chi:=\{S\} ; \mathcal{M}:=\left\{\left\langle a, M_{a}\right\rangle: a \in\right.$ Act $\} ;$ SplCnd $:=\mathcal{M} ;$

While $\operatorname{SplCnd} \neq \emptyset$ do

(1) choose some step class $\langle a, M\rangle$ of $S p l C n d$ and remove $\langle a, M\rangle$ from SplCnd;

(2) (* Computation of $\chi_{\text {new }}:=\operatorname{Ref}(\chi, a, M) *$ )

$P:=\operatorname{Pre}^{\text {del }}(a, M) ; \chi_{\text {new }}:=\emptyset ; N e w B l:=\emptyset$

For all $B \in \chi$ do

$$
B^{\prime}:=B \cap P ; B^{\prime \prime}:=B \backslash B^{\prime} ; \chi_{\text {new }}:=\chi_{\text {new }} \cup\left\{B^{\prime}, B^{\prime \prime}\right\} \backslash\{\emptyset\} ;
$$

If $\emptyset \neq B^{\prime} \neq B$ then

$$
\begin{aligned}
& \text { If }\left|B^{\prime}\right| \leq\left|B^{\prime \prime}\right| \text { then } C^{\prime}:=B^{\prime} \text { else } C^{\prime}:=B^{\prime \prime} ; \\
& N e w B l:=N e w B l \cup\left\{C^{\prime}\right\} ;
\end{aligned}
$$

(3) (* Computation of $\mathcal{M}_{\text {new }}:=\mathcal{M}_{\chi_{\text {new }}} *$ )

$\mathcal{M}_{\text {new }}:=\emptyset$;

For all $\langle b, N\rangle \in \mathcal{M}$ do

$$
\begin{aligned}
& \mathcal{N}:=\operatorname{Split}(\langle b, N\rangle, N e w B l) ; \mathcal{M}_{\text {new }}:=\mathcal{M}_{\text {new }} \cup \mathcal{N} ; \\
& \text { If }|\mathcal{N}| \geq 2 \text { then SplCnd }:=\operatorname{SplCnd} \cup \mathcal{N} ;
\end{aligned}
$$

(4) $\chi:=\chi_{\text {new }} ; \mathcal{M}:=\mathcal{M}_{\text {new }}$;

Return $\chi$.

Fig. 3. Algorithm for the (strict) normed bisimulation equivalence classes

Lemma 1. $\operatorname{Pre}_{b}^{\text {del }}(a, M)$ can be computed in time $\mathcal{O}\left(m_{\tau} n\right)$.

Proof. $\operatorname{Pre}_{b}^{\text {del }}(a, M)$ is the least subset of $S$ satisfying the conditions (BD0), (BD1) and (BD2). The standard iterative method for computing the least fixed point of a monotonic set-valued operator leads to the following method for computing $\operatorname{Pre}_{b}^{d e l}(a, M)$. We consider the directed graph $G_{b}^{d e l}(a, M)=(V, E)$ with the vertex set $V=S \cup M_{\tau}$ and the edge set $E=\left\{(\nu, s) \in M_{\tau} \times S: s \stackrel{\tau}{\longrightarrow} \nu\right\}$ $\cup\left\{(s, \nu) \in S \times M_{\tau}: s \in S u p p(\nu)\right\}$. We assume a representation of $G_{b}^{d e l}(a, M)$ by adjacency lists and write $E(\cdot)$ to denote the adjacency list of $(\cdot)$. We use the algorithm shown in Fig. 4 to compute the set $\operatorname{Pre}_{b}^{d e l}(a, M)$. For any $\nu \in N$, we use a counter $c(\nu)$ for the number of states $t \in \operatorname{Supp}(\nu)$ where the condition $t \in \operatorname{Pre}_{b}^{d e l}(a, M)$ is not yet verified. $N_{0}$ collects all $\nu$ where $c(\nu)=0$, i.e. $\operatorname{Supp}(\nu) \subseteq \operatorname{Pre}_{b}^{\text {del }}(a, M)$. Hence, if $\nu_{0} \in N_{0}$ and $s \stackrel{\tau}{\longrightarrow} \nu_{0}$ then we may insert $s$ into $\operatorname{Pre}_{b}^{d e l}(a, M)$. Clearly, this method can be implemented in time $\mathcal{O}\left(m_{\tau} n\right)$.

Lemma 2. $\operatorname{Pre}_{u b}^{d e l}(a, M)$ can be computed in time $\mathcal{O}\left(m_{\tau} n\right)$.

Proof. To compute $\operatorname{Pre}_{u b}^{d e l}(a, M)$ we suggest a graph-theoretical method which is based on the following observation. $\operatorname{Pre}_{u b}^{d e l}(a, M)$ is the least subset of $S$ which contains $\operatorname{Pre}^{\leq 1}(a, M)=\operatorname{Pre}^{0}(a, M) \cup \operatorname{Pre}^{1}(a, M)$ (where $\operatorname{Pre}^{0}(\tau, M)=\{s$ : $\left.\mu_{s}^{1} \in M\right\}, \operatorname{Pre}^{0}(a, M)=\emptyset$ if $a$ is visible and $\left.\operatorname{Pre}^{1}(a, M)=\{s: s \stackrel{a}{\longrightarrow} M\}\right)$ and 
Compute the adjacency lists $E(\cdot)$ of the graph $G_{b}^{\text {del }}(a, M)$;

If $a=\tau$ then $\operatorname{Pre}_{b}^{d e l}(a, M):=\left\{s: \mu_{s}^{1} \in M\right\}$ else $\operatorname{Pre}_{b}^{d e l}(a, M):=\emptyset$;

$N_{0}:=\emptyset$ and $c(\nu):=|\operatorname{Supp}(\nu)|$ for all $\nu \in N$;

For all $s \in S$ where $s \stackrel{a}{\longrightarrow} \mu$ for some $\mu \in M$ do:

$\operatorname{Pre}_{b}^{d e l}(a, M):=\operatorname{Pre}_{b}^{d e l}(a, M) \cup\{s\} ;$

For all $\nu \in E(s)$ do

$$
\begin{aligned}
& c(\nu):=c(\nu)-1 ; \\
& \text { If } c(\nu)=0 \text { then } N_{0}:=N_{0} \cup\{\nu\} ;
\end{aligned}
$$

While $N_{0} \neq \emptyset$ do

choose some $\nu_{0} \in N_{0}$ and put $N_{0}:=N_{0} \backslash\left\{\nu_{0}\right\}$;

For all $s \in E\left(\nu_{0}\right) \backslash \operatorname{Pre}_{b}^{\text {del }}(a, M)$ do

$$
\begin{aligned}
& \operatorname{Pre}_{b}^{\text {del }}(a, M):=\operatorname{Pre}_{b}^{d e l}(a, M) \cup\{s\} ; \\
& \text { For all } \nu \in E(s) \backslash N_{0} \text { do } \\
& \qquad \begin{array}{l}
c(\nu):=c(\nu)-1 ; \\
\text { If } c(\nu)=0 \text { then } N_{0}:=N_{0} \cup\{\nu\} ;
\end{array}
\end{aligned}
$$

Return $\operatorname{Pre}_{b}^{\text {del }}(a, M)$.

Fig. 4. Algorithm for computing $\operatorname{Pre}_{b}^{\text {del }}(a, M)$

satisfies the following condition. Whenever $C \subseteq S$ such that for any $s \in C$ there is a finite path $s=s_{0} \stackrel{\tau, \nu_{0}}{\longrightarrow} s_{1} \stackrel{\tau, \nu_{1}}{\longrightarrow} \ldots \stackrel{\tau, \nu_{l-1}}{\longrightarrow} s_{l} \stackrel{\tau, \nu_{l}}{\longrightarrow} t$ where $s_{1}, \ldots, s_{l} \in C, s_{i} \stackrel{\tau}{\longrightarrow} \nu_{i}$ with $\operatorname{Supp}\left(\nu_{i}\right) \subseteq C \cup \operatorname{Pre}_{u b}^{d e l}(a, M), i=0,1, \ldots, l$, and $t \in \operatorname{Pre}_{u b}^{d e l}(a, M)$ then $C \subseteq$ $\operatorname{Pre}_{u b}^{d e l}(a, M)$. On the basis of this characterization, we compute $\operatorname{Pre}_{u b}^{d e l}(a, M)$ as follows. We start with $\operatorname{Pre}_{u b}^{d e l}(a, M)=\operatorname{Pre}^{\leq 1}(a, M)$ Then, we successively add all states of a set $C$ satisfying the above condition which can be reformulated by means of the strongly connected components (SCCs) in a certain directed graph. We consider the directed graph $G_{u b}^{\text {del }}(a, M)=(V, E)$ where the vertex set $V$ is given by $V=\left\{(s, \nu): \nu \in \operatorname{Steps}_{\tau}(s), s \notin \operatorname{Pre}^{\leq 1}(a, M)\right\} \cup \operatorname{Pre}^{\leq 1}(a, M)$ and the edge set is $E=\left\{\left\langle(s, \nu),\left(s^{\prime}, \nu^{\prime}\right)\right\rangle: s \in \operatorname{Supp}\left(\nu^{\prime}\right)\right\} \cup\left\{\left\langle u,\left(s^{\prime}, \nu^{\prime}\right)\right\rangle\right.$ : $\left.u \in \operatorname{Pre}^{\leq 1}(a, M) \cap \operatorname{Supp}\left(\nu^{\prime}\right)\right\}$. First we compute the SCCs of $G=G_{u b}^{\text {del }}(a, M)$ and a topological sorting $C_{1}, \ldots, C_{r}$ on them. The singleton sets $\{u\}$ where $u \in \operatorname{Pre}^{\leq 1}(a, M)$ are bottom SCCs (BSCCs) in $G$. Thus, we may assume that there is some $h$ such that $\operatorname{Pre}^{\leq 1}(a, M)=C_{1} \cup \ldots \cup C_{h}$ and $C_{i} \subseteq V \backslash \operatorname{Pre}^{\leq 1}(a, M)$, $i=h+1, \ldots, r$. We start with $\operatorname{Pre}_{u b}^{d e l}(a, M)=\operatorname{Pre}^{\leq 1}(a, M)$. For $i=h+1, \ldots, r$, if $C_{i}$ is not a BSCC (i.e. $\left\{j: C_{j} \rightarrow_{G} C_{i}\right\} \neq \emptyset$ ) and all states of a predecessor SCC $C_{j}$ of $C_{i}$ belong to $\operatorname{Pre}_{u b}^{d e l}(a, M)$ then we insert the states of $C_{i}$ into $\operatorname{Pre}_{u b}^{d e l}(a, M)$. Clearly, $G=G_{u b}^{d e l}(a, M)$ has $\mathcal{O}\left(m_{\tau}+n\right)$ vertices and $\mathcal{O}\left(m_{\tau} n\right)$ edges. Hence, this method can be implemented in time and space $\mathcal{O}\left(m_{\tau} n\right)$. 
Proposition 4. Ranging over all iterations, the computation of the step partitions $\mathcal{M}_{\text {new }}$ in step (3) of Figure 3 takes $\mathcal{O}(m n(\log m+\log n))$ time.

Proof. For calculating $\operatorname{Split}\left(\left\langle b, N^{\prime}\right\rangle, C^{\prime}\right)$ we may apply a technique (similar to the one suggested in [3]) which generates an ordered balanced tree (e.g. AVL tree) by successively inserting the values $\nu\left[C^{\prime}\right], \nu \in N^{\prime}$, possibly creating new nodes and performing the necessary rebalancing steps. Any node $v$ in this tree is labelled by a key value $v$.key (which is one of the probabilities $\nu\left[C^{\prime}\right]$ for one or more $\nu \in N^{\prime}$ ) and a subset $v$.distr of $N^{\prime}$. Then, $\operatorname{Split}\left(\left\langle b, N^{\prime}\right\rangle, C^{\prime}\right)$ is the set of pairs $\langle b, v$.distr $\rangle$ where $v$ is a node in the final tree. The construction of the tree causes the cost $\mathcal{O}\left(\left|N^{\prime}\right| \log \left|N^{\prime}\right|\right)$ as for any $\nu \in N^{\prime}$ we traverse a tree of height $\leq \log \left|N^{\prime}\right|$. For fixed $\nu$ and $C^{\prime}$, the computation of the values $\nu\left[C^{\prime}\right]$ can be implemented in time $\mathcal{O}\left(\left|C^{\prime}\right|\right)$. Thus, for any call of the procedure $\operatorname{Split}\left(\left\langle b, N^{\prime}\right\rangle, C^{\prime}\right)$ the time spent for computing the values $\nu\left[C^{\prime}\right]$ is $\mathcal{O}\left(\left|N^{\prime}\right| \cdot\left|C^{\prime}\right|\right)$ where we range over all $\nu \in N^{\prime}$. Summing up over all step classes $\langle b, N\rangle$ in the current step partition $\mathcal{M}$, any $C^{\prime} \in A l l N e w B l$ causes the cost $\mathcal{O}\left(m \log m+m\left|C^{\prime}\right|\right)$. (ii) and (iii) yield the time complexity $\mathcal{O}(m n(\log m+\log n))$ for all $\operatorname{Split}(\cdot)$ operations together.

\section{Conclusion}

We introduced two notions of bisimulation equivalence in probabilistic systems (with non-determinism) that abstract from internal computations. We presented polynomial-time algorithms that compute the quotient spaces and briefly discussed other important issues (soundness for establishing linear time properties and compositionality). Thus, our notion of bisimulation equivalence yields an alternative to the weak and branching bisimulations of [36 37]. Although the equivalences à la [36/37] are the natural probabilistic counterpart to weak/branching bisimulation equivalence in LTSs [28,3116], their definitions are rather complicated and the decidability is still an open problem. We argue that the definitions of our equivalences - which rely on the rather intuitive concept of norm functions à la [17] - are comparatively simple. Moreover, the use of norm functions in the definition of our equivalences allows for a characterization of the equivalence classes by means of graph-theoretical criteria which served as basis for our algorithm that computes the equivalence classes. In particular, the characterization of the delay predecessor predicates that we used in the proofs of Lemmatas 1 and 2 can easily be rewritten as terms of the relational mu-calculus. It would be interesting if our ideas can be combined with the techniques of [8,12] for computing the bisimulation equivalence in LTSs with a BDD-based model checking algorithm for the relational mu-calculus seems to get a symbolic technique that might combat the state explosion problem for PLTSs.

In this paper (where we mainly treated the issue of decidability) we restrict our attention to finite systems. However, norm functions and the derived notions of bisimulations can also be defined for infinite systems 12 We believe that, as in

$\overline{12}$ For our purposes, it was sufficient to consider the natural numbers as range of the norm functions. The framework of [17] also covers infinite, possibly uncountable, 
the non-probabilistic case, in many applications, it is quite simple to "guess" a norm function and then to check (e.g. by hand) whether it fulfills the necessary conditions. Further on, the concept of norm functions can also serve as basis for simulation preorders that abstracts from internal moves and is computable in finite systems. Further details about normed simulations can be found in [6] and the forthcoming work 39 .

Acknowledgements: The authors would like to thank Frits Vaandrager and Holger Hermanns for many helpful comments.

\section{References}

1. P. d'Argenio, H. Hermanns, J. Katoen: On Generative Parallel Composition, Proc. PROBMIV'98, ENTCS, Vol. 21, 1999.

2. J. Baeten, J. Bergstra, S. Smolka: Axiomatizing Probabilistic Processes: ACP with Generative Probabilities, Inf. and Comp., Vol. 122, pp 234-255, 1995.

3. C. Baier, B. Engelen, M. Majster-Cederbaum: Deciding Bisimilarity and Similarity for Probabilistic Systems, to appear in Journ. of Computer and System Sc., 2000.

4. C. Baier, H. Hermanns: Weak Bisimulation for Fully Probabilistic Processes, Proc. CAV'97, LNCS 1254, pp 119-130, 1997.

5. C. Baier, M. Kwiatkowska: Domain Equations for Probabilistic Processes, to appear in Mathematical Structures in Computer Science, 2000.

6. C. Baier, M. Stoelinga: Probabilistic Bisimulation and Simulation: Decidability, Complexity and Compositionality, Techn. Report, University Nijmegen, 1999.

7. T. Bolognesi, S. Smolka: Fundamental Results for the Verification of Observational Equivalence: a Survey, Proc. Protocol Specification, Testing and Verification, Elsevier Science Publishers, IFIP, pp 165-179, 1987.

8. J. Burch, E. Clarke, K. McMillan, D. Dill, L. Hwang: Symbolic Model Checking: $10^{20}$ States and Beyond, Inform. and Computation, Vol. 98 (2), pp 142-170, 1992.

9. L. Christoff: Specification and Verification Methods for Probabilistic Processes, Ph. D. Thesis, Uppsala University, 1993.

10. L. Christoff, I. Christoff: Efficient Algorithms for Verification of Equivalences for Probabilistic Processes, Proc. CAV'91, LNCS 575, pp 310-321,1991.

11. T. Cormen, C. Leiserson, R. Rivest: Introd. to Algorithms, McGraw Hill, 1996.

12. R. Enders, T. Filkorn, D. Taubner: Generating BDDs for Symbolic Model checking in CCS, Distributed Computing, Vol. 6, pp 155-164, 1993.

13. A. Giacalone, C. Jou, S. Smolka: Algebraic Reasoning for Probabilistic Concurrent Systems, Proc. IFIP TC2 Working Conf. on Prog. Concepts and Methods, 1990.

14. R. van Glabbeek: The Linear Time - Branching Time Spectrum II: The Semantics of Sequential Systems with Silent Moves, extended abstract, Proc. CONCUR'93, LNCS 715, pp 66-81, 1993.

15. R. van Glabbeek, S. Smolka, B. Steffen, C. Tofts: Reactive, Generative, and Stratified Models for Probabilistic Processes, Proc. LICS'90, pp 130-141, 1990.

state spaces and allows for arbitrary well-founded sets as range of norm functions. We argue that these ideas can also be used to handle PLTSs of arbitrary size. 
16. R. van Glabbeek, W. Weijland: Branching Time and Abstraction in Bisimulation Semantics, Journal of the ACM, Vol. 43(3), pp 555-600,1996.

17. D. Griffioen, F. Vaandrager: Normed Simulations, Proc. CAV'98, LNCS 1427, pp 332-344, 1998.

18. H. Hansson: Time and Probability in Formal Design of Distributed Systems, in Real-Time Safety Ciritical Systems, Vol. 1, Elsevier, 1994.

19. H. Hansson, B. Jonsson: A Calculus for Communicating Systems with Time and Probabilities, Proc. IEEE Real-Time Systems Symposium, IEEE Computer Society Press, pp 278-287, 1990.

20. T. Huynh, L. Tian: On some Equivalence Relations for Probabilistic Processes, Fundamenta Informaticae, Vol. 17, pp 211-234, 1992.

21. B. Jonsson, C. Ho-Stuart, W. Yi: Testing and Refinement for Nondeterministic and Probabilistic Processes, Proc. FTRTFT'94, LNCS 863, pp 418-430, 1994.

22. B. Jonsson, K.G. Larsen: Specification and Refinement of Probabilistic Processes, Proc. LICS'91, pp 266-277, 1991.

23. B. Jonsson, W. Yi: Compositional Testing Preorders for Probabilistic Processes, Proc. LICS'95, pp 431-443, 1995.

24. C. Jou, S. Smolka: Equivalences, Congruences and Complete Axiomatizations for Probabilistic Processes, Proc. CONCUR'90, LNCS 458, pp 367-383, 1990.

25. P. Kannelakis, S. Smolka: CCS Expressions, Finite State Processes and Three Problems of Equivalence, Information and Computation, Vol. 86, pp 43-68, 1990.

26. K. Larsen, A. Skou: Bisimulation through Probabilistic Testing, Information and Computation, Vol. 94, pp 1-28, 1991.

27. K. Larsen, A. Skou: Compositional Verification of Probabilistic Processes, Proc. CONCUR'92, LNCS 630, pp 456-471, 1992.

28. R. Milner: A Calculus of Communicating Systems, LNCS 92, 1980.

29. R. Milner: Communication and Concurrency, Prentice Hall, 1989.

30. R. Paige, R. Tarjan: Three Partition Refinement Algorithms, SIAM Journal of Computing, Vol. 16, No. 6, pp 973-989, 1987.

31. D. Park: Concurrency and Automata on Infinite Sequences, Proc. 5th GI Conference, LNCS 104, pp 167-183, 1981.

32. A. Philippou, O. Sokolsky, I. Lee: Weak Bisimulation for Probabilistic Systems, submitted for publication.

33. M. Puterman: Markov Decision Processes, John Wiley and Sons, 1994.

34. R. Segala: Modeling and Verification of Randomized Distributed Real-Time Systems, Ph.D.Thesis, Massachusetts Institute of Technology, 1995.

35. R. Segala: A Compositional Trace-Based Semantics for Probabilistic Automata, Proc. CONCUR'95, LNCS 962, pp 234-248, 1995.

36. R. Segala, N. Lynch: Probabilistic Simulations for Probabilistic Processes, Proc. CONCUR'94, LNCS 836, pp 481-496, 1994.

37. R. Segala, N. Lynch: Probabilistic Simulations for Probabilistic Processes, Nordic Journal of Computing, Vol. 2 (2), pp 250-273, 1995.

38. M. Stoelinga, F. Vaandrager: Root Contention in IEEE 1394, Proc. ARTS'99, LNCS 1601, pp 53-74, 1999.

39. M. Stoelinga: Hypernorms for Probabilistic Systems, in preparation, 2000. 
40. W. Weijland: Synchrony and Asynchrony in Process Algebra, Ph.D.Thesis, Univesity of Amsterdam, 1989.

41. M. Vardi: Automatic Verification of Probabilistic Concurrent Finite-State Programs, Proc. FOCS'85, pp 327-338, 1985.

42. W. Yi, K. Larsen: Testing Probabilistic and Nondeterministic Processes, Proc. Protocol, Specification, Testing, Verification XII, pp 47-61, 1992.

43. W. Yi: Algebraic Reasoning for Real-Time Probabilistic Processes with Uncertain Information, Proc. FTRTFT'94, LNCS 863, pp 680-693, 1994. 